\title{
Caractéristiques végétales, typologie et fonctions des bois sacrés au Burkina Faso
}

\author{
Salfo SAVADOGO ${ }^{1 *}$, Augustin KABORE ${ }^{2}$ et Adjima THIOMBIANO $^{3}$ \\ ${ }^{I}$ Département Substances Naturelles (DSN), Institut de Recherche en Sciences Appliquées et Technologies \\ (IRSAT), Centre National de la Recherche Scientifique et Technologiques (CNRST), \\ 03 BP 7047 Ouagadougou 03, Burkina Faso. \\ ${ }^{2}$ Direction Générale de l'Economie Verte et du Changement Climatique (DGEVCC), \\ 03 BP 7044 Ouagadougou 03, Burkina Faso. \\ ${ }^{3}$ Laboratoire de Biologie et Ecologie Végétales (LaBev), Université Ouaga I Pr. Joseph Ki-Zerbo, (UO I/Pr. \\ JKZ), Unité de Formation et de Recherche en Sciences de la vie et de la Terre (UFR/SVT), 09 BP 848 \\ Ouagadougou 09, Burkina Faso. \\ *Auteur correspondant ; E-mail : salfosava@gmail.com; Tél : (+226) 78148774
}

\section{REMERCIEMENTS}

Nous Remercions vivement le Projet BIOTA (Biodiversity Monitoring Transect Analysis) pour avoir financé la collecte de données de cet article.

\section{RESUME}

Au Burkina Faso, les bois sacrés sont des îlots de végétations conservés et gérés par les autorités coutumières. Pour comprendre les caractéristiques végétales, la typologie et les fonctions de ces bois sacrés, une étude a été réalisée à l'aide d'enquêtes ethnobotaniques auprès de 32 groupes ethniques suivie de relevés botaniques sur le terrain. Ces études montrent qu'au Burkina Faso: selon l'incarnation du bois, il y a les bois fétiches, les bois cimetières, les bois fétiches-cimetières, les bois de divinités et les bois hantés ; suivant la propriété, on distingue les bois personnels, les bois familiaux, les bois de quartier, les bois communautaires et les bois de sociétés secrètes. Ils sont dominés par des espèces telles que Acacia erythrocalyx Brenan, Anogeissus leiocarpa (DC.) Guill. \& Perr., Combretum micranthum G. Don, Combretum nigricans Lepr. ex Guill. \& Perr., Balanites aegyptiaca (L.) Del., Khaya senegalensis (Desv.) A. Juss., Isoberlinia doka Craib \& Stapf, Maranthes polyandra (Benth.) Prance, Daniellia oliveri (Rolfe) Hutch. \& Dalz., Burkea africana Hook., Monotes kerstingii Gilg. Les bois sacrés assurent aux populations, plusieurs fonctions: écologique, cultuelle, socio-culturelle, magique et mixte. Parmi les bois sacrés, seuls ceux qui suscitent la crainte à l'endroit de la population sont intégralement protégés.

(C) 2017 International Formulae Group. All rights reserved.

Mots clés: Bois sacré, enquête ethnobotanique, caractéristiques végétales, fonctions, typologie, Burkina Faso. 


\title{
Plant characteristics, typology and functions of sacred woodlands in Burkina Faso
}

\begin{abstract}
In Burkina Faso, sacred woodlands are islands of vegetation preserved and managed by the customary authorities. To understand the plant characteristics, the typology and the functions of these sacred woodlands, a study has been carried out using ethnobotanical investigation along 32 ethnic groups and floristic surveys. Surveys show that in Burkina Faso: According to the incarnation of the sacred woodland, there are the fetish sacred woodlands, the cemetery sacred woodlands, the fetish-cemeteries sacred woodlands, the sacred woodlands of deities and the haunted sacred woodlands; Depending on the property, we can distinguish personal sacred woods, family sacred woodlands, neighborhood sacred woodlands, community sacred woodlands and woodlands of secret societies. They are dominated by the species such as Acacia erythrocalyx Brenan, Anogeissus leiocarpa (DC.) Guill. \& Perr., Combretum micranthum G. Don, Combretum nigricans Lepr. ex Guill. \& Perr., Balanites aegyptiaca (L.) Del., Khaya senegalensis (Desv.) A. Juss., Isoberlinia doka Craib \& Stapf, Maranthes polyandra (Benth.) Prance, Daniellia oliveri (Rolfe) Hutch. \& Dalz., Burkea africana Hook., Monotes kerstingii Gilg. The sacred woodlands provide to the populations several functions: ecological, religious, socio-cultural, magical and mixed. Among the sacred woodlands, only those which arouse fear among the population are well protected.

(C) 2017 International Formulae Group. All rights reserved.
\end{abstract}

Keywords: Sacred woodland, ethnobotanical investigation, plant characteristics, functions, typology, Burkina Faso.

\section{INTRODUCTION}

Pour beaucoup de sociétés africaines, la terre et la végétation restent durablement en lien avec les ancêtres qui les ont travaillées auparavant. Maintenir de bons rapports avec les ancêtres est considéré comme essentiel pour le bon développement des cultures et du village (Garcia et al., 2006 ; Langewiesche, 2006 ; Kaboré, 2010; Savadogo et al., 2011; Savadogo, 2013). C'est en ayant conscience de cette situation que les sociétés se rendent dans les forêts pour vouer des cultes à leurs ancêtres. Tous les courants spirituels incluent, à leur façon, des préceptes de respect de la nature (WWF-France, 2003 ; Fournier, 2011 ; Savadogo et al., 2011 ; Savadogo, 2013 ; Ali et al., 2014 ; Olohou et al., 2016). La forêt dans son ensemble est perçue comme un espace généreux, parce qu'elle nourrit et qu'elle offre la perspective d'une guérison grâce à ses produits. Aussi, elle est appréhendée comme un espace qui effraie, un espace où vivent les génies, les animaux sauvages et les malfaiteurs (Langewiesche, 2006 ; Malan, 2009 ; Matthieu, 2010).
Les sites sacrés sont vénérés par des populations autochtones et traditionnelles et constituent un élément fondamental de leur territoire, apportant des avantages importants aux communautés locales, nationales et mondiales. De façon générale, ils remplissent plusieurs fonctions dont les plus importantes sont les fonctions socioculturelles et religieuses. Selon Houngnihin (2005), la consécration des forêts aux divinités ancestrales (forêts sacrées) est l'une des techniques endogènes de conservation des ressources naturelles et de protection de la nature pour en constituer des jardins botaniques et écologiques. Selon Savadogo (2013), les politiques d'aménagement banalisent cependant les lieux sacrés et les fonctions traditionnelles de la forêt (sécurité alimentaire, pharmacologie, technologie, pédagogie). De ce qui précède, il importe de trouver les voies et moyens pour soutenir ces institutions sociales dans la préservation de la tradition. $\mathrm{Ce}$ qui augmenterait leur engouement à la protection des lieux de culte, sanctuaire de la diversité biologique (Kokou et Kokutse, 2007). 
Les aspects et les statuts socioculturels des bois sacrés ont été abordés par des auteurs, dans certaines régions du pays (Bognounou et al., 2001; Juhé-Beaulaton et al., 2005 ; Traoré, 2007; Savadogo, 2008; Savadogo et al., 2010; Savadogo et al., 2011). Thiombiano (2005), décrivant les pratiques culturelles rattachées à certaines Combrétacées en pays Gourmantché, a observé 8 bois sacrés dans lesquels ont lieu des sacrifices rituels. Fournier (2011) a abordé l'impact des pratiques culturelles dans la conservation des ressources ligneuses en pays Bwaba.

L'une des limites des études ethnobotaniques antérieures découle du fait qu'elles n'aient pas pu couvrir toute l'étendue $\mathrm{du}$ territoire du Burkina Faso; d'où la nécessité de mener une étude sur toute l'étendue du pays en prenant en compte plusieurs groupes ethniques. L'étude se justifie par le fait que les différentes fonctions des bois sacrés présentent l'avantage de motiver la population locale à les préserver.

L'objectif de cette étude est d'avoir une base de données sur les bois sacrés du Burkina Faso notamment leurs distributions spatiales, leurs caractéristiques végétales, leur typologie et leurs importances pour les populations. De façon spécifique, l'étude vise à :

- décrire les caractéristiques végétales des bois sacrés ;

- répertorier les différentes fonctions des bois sacrés ;

-faire une classification typologique et établir des cartes de distribution de ces bois sacrés ;

\section{MATERIEL ET METHODES Milieu d'étude}

L'étude a couvert 330 villages, 40 provinces et 13 régions du Burkina Faso. Les groupes ethnico-lingustiques concernés par l'étude étaient au nombre de 32 (Mossé, Gourmantché, Samo, Bisa, Bwaba, Gourousi, Peulh, Bella, Haoussa, Bolon, Dogossé, Bobo, Sénoufo, Gan, Djan, Pougouli, Birifor, Dagara, Lobi, Dagara-Dioula, Touni, Fulsé, Jaossé, Yana, Marka, Turka, Tiéfo, Karaboro, Sissala, Dogon, Toussian, Goin). Chaque groupe ethnique occupe une position géographique bien donnée (Figure 1).

\section{Collecte des données}

Centrée sur des terroirs villageois et des groupes ethniques choisis de façon raisonnée, la recherche de terrain a associé une enquête par questionnaire auprès de 3265 autochtones d'âges supérieurs à cinquante (50) ans.

Dans chaque localité, les personnes cibles ont été les autorités coutumières, les responsables des fétiches et les notables. Toutefois, certains jeunes affiliés à ces catégories de personnes ont été interviewés et ont pu fournir des informations qui ont été validées par les mêmes autorités. Les 3265 personnes enquêtées appartiennent à 32 groupes ethniques choisis sur la base de la vivacité de la tradition et des coutumes (ampleur des sacrifices qui y sont liés, capacités des communautés à maintenir le bois sacré existant, constances des évènements rituels de nature à encourager la conservation des bois sacrés,...). Le nombre moyen de personnes enquêtées par groupe ethnique était de 100.

Les entretiens ont été faits avec des groupes d'individus, à l'aide d'un guide de terrain. Le nombre moyen de personnes par focus groupe est de 15. Des relevés botaniques et des observations directes ont été réalisés dans les bois sacrés afin d'inventorier les espèces végétales et d'apprécier les différents types de formations végétales en leurs seins. Ces méthodes ont été déjà utilisées par certains auteurs (Garba et al., 2017; Seydou et al., 2017) et ont donné des résultats satisfaisants. Les coordonnées des centres des bois ont été prises pour leur localisation ultérieure sur des cartes typologiques. Pour ce faire, des GPS de plusieurs types (Garming 12, Garming 72, $60 \mathrm{Cx}$ ) ont été utilisés. Les points sont enregistrés directement dans les GPS puis reportés sur une fiche préalablement élaborée.

\section{Traitement des données}

Les données de l'enquête ont d'abord été dépouillées manuellement puis traitées sur 
Excel 2010 et sur JMP 12. Les proportions des différents types de bois sacrés et leurs fonctions ont été calculées et illustrées sous forme de diagrammes circulaires. Les différents types de bois sacrés et leur appartenance ethnique ont été synthétisés sous forme de tableaux.

Les résultats sont présentés sous forme de diagramme circulaire. Des cartes de distribution des différentes catégories de bois ont été élaborées. Pour ce faire, les coordonnées géographiques sont d'abord transférées du GPS vers Excel avant d'être transporté sur Arc view Gis 3.2a. Toutes les données floristiques ont été synthétisées dans un tableur Excel sous forme d'une matrice de relevés $\mathrm{x}$ espèces. La matrice a été convertie en données de présence-absence et soumise à une série d'analyses multivariées au moyen de CANOCO (CANOnical Comminity Ordination) version 4.5 pour la recherche des espèces dominantes dans les bois sacrés. Les types de formations végétales ont été déterminés sur le terrain à travers une estimation directe.

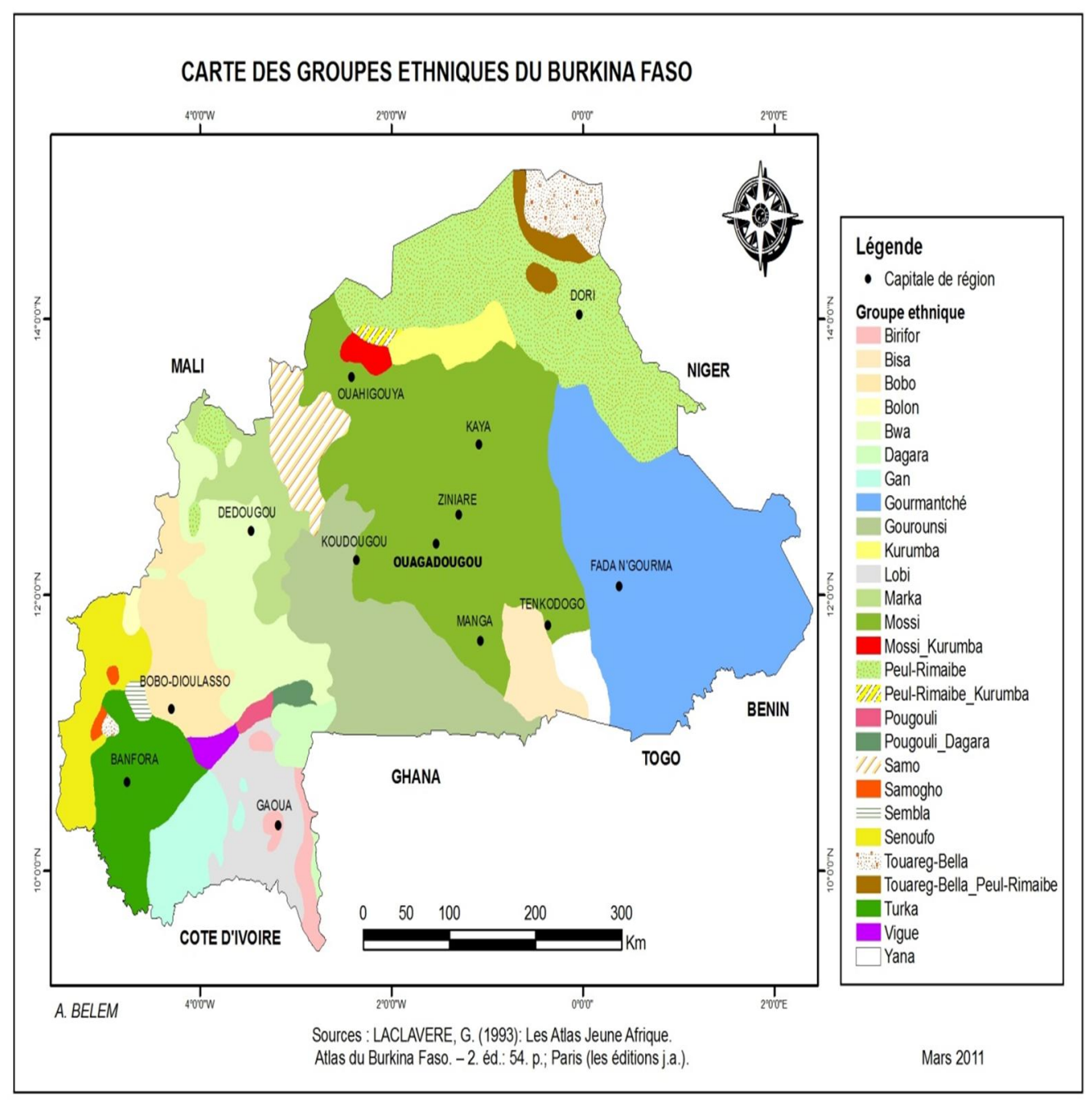

Figure 1: Carte des groupes ethnico-lingustiques du Burkina Faso. (Laclavere, 1993: Les Atlas Jeune Afrique). 


\section{RESULTATS}

\section{Caractéristiques végétales des bois sacrés}

Les bois sacrés sont en général des formations physionomiquement denses, isolées, le plus souvent situées à quelques encablures des habitations. Du sahel jusqu'au Nord soudanien, ils sont identifiables par l'abondance d'espèces remarquables comme Acacia erythrocalyx et Combretum micranthum qui forment des fourrés presqu'impénétrables. On y observe quelquefois des pieds de Khaya senegalensis et de Anogeissus leiocarpa qui émergent de la strate arbustive. Des formations monospécifiques à Balanites aegyptiaca sont également rencontrées dans ces secteurs. Les bois sacrés sont en général des steppes, des fourrés, des savanes arbustives, des savanes arborées, des savanes boisées, des forêts claires et des galeries forestières. Les fourrés ( $20 \%$ des bois sacrés) sont des formations basses difficilement pénétrables. Ils sont de trois types: les fourrés monospécifiques à Acacia erythrocalyx, à Combretum micranthum et les fourrés plurispécifiques constitués essentiellement par ces deux espèces et quelques espèces ubiquistes. Les fourrés sont très fréquents dans le secteur subsahélien. Les savanes arbustives sont les plus nombreuses de toutes les formations ( $50 \%$ des bois sacrés). Elles sont très souvent des peuplements monospécifiques des espèces telles Combretum micranthum, Combretum nigricans et Anogeissus leiocarpa. On y trouve également quelques peuplements plurispécifiques. Les savanes arborées sont surtout concentrées dans le secteur nord soudanien et représentent $20 \%$ des bois sacrés. Les savanes boisées et les forêts sont plus fréquentes dans le secteur sud soudanien $(10 \%$ des bois sacrés). Les espèces couramment rencontrées dans les bois sacrés de ce secteur sont Khaya senegalensis, Anogeissus leiocarpa, Isoberlinia doka, Maranthes polyandra, Daniellia oliveri, Entada africana, Burkea africana, Monotes kerstingii.

\section{Catégorisation des bois sacrés Selon l'incarnation ou la représentation}

Les sites sacrés sont subdivisés en bois cimetière, bois fétiche, bois fétiche-cimetière, bois de divinité et bois hantés en tenant compte de leur représentation ou leur incarnation (Figure 2). Ces différentes catégories appartiennent à des groupes ethniques bien déterminés (Tableau 1).

Les bois cimetières abritent les tombeaux physiques ou mythiques des personnes ayant subi une mort brutale ou accidentelle (généralement par la foudre, les accidents de circulation, les brûlures de feu, les noyades, les maladies contagieuses et épidémiques), des femmes qui meurent en état de grossesse ou durant l'accouchement, des rois, des reines et souvent des petits enfants.

Ces bois sont très fréquents en société Mossé, Gourmantché, Fulsé, Yana, Toussian et Bissa (Tableau 1). Ils bénéficient d'une protection intégrale à cause de la crainte et du respect dû aux morts.

Les bois fétiches abritent les fétiches du village, des quartiers, des clans, etc. Les fétiches sont matérialisés par des objets de plusieurs types : roches granitiques ou latéritiques, des cauris, outils en terre cuite (jarre, canari, marmite), etc. La protection des bois fétiches n'est pas intégrale comme le cas des bois cimetières. Malgré l'effort des autorités en charge de leur protection, on enregistre en effet, des cas d'exploitation frauduleuse des ressources et de divagation d'animaux. Dans tous les groupes sociaux où la religion traditionnelle demeure, il y a des bois fétiches. Ils sont repartis dans toutes les régions du Burkina Faso (Figure 3).

Les bois fétiche-cimetières abritent à la fois des fétiches (du village ou du quartier) et des cimetières des rois ou des chefs de terre. Ils sont également protégés à cause de la présence des cimetières. Ces bois sont uniquement rencontrés chez les Mossé, les Fulsé et les Gourmantché.

Les bois de divinités abritent les divinités claniques ou personnelles. Les 
divinités sont faites en statuette de bois ou de vase en terre cuite, grande de taille et hautement considérées et vénérées. Les bois de divinité sont surtout rencontrés chez les Mossé, les Gourmantché, les Bwaba, les Gouroussi, les Samo, les Lobi, les Birifor, les Dagara, les Gan et les Pougouli. Ils ne sont pas intégralement protégés car ils ne suscitent aucune crainte à l'endroit des populations riveraines.

Les bois hantés sont conçus par l'imaginaire social reliant des ensembles d'éléments symboliques et mystiques à une partie de la végétation. La société dans ses attitudes leur voue des égards particuliers parce qu'ils incarnent l'existence d'êtres (génies et démons) dont la puissance est supérieure à celle de l'homme. Ils sont très fréquents chez les Mossé, les Dafing, les Fulsé, les Gouroussi, les Bwaba, les Bobo, les Gourmantché, les Bolon, les Yana et les Bissa. Ils bénéficient d'une autoprotection intégrale à cause de la crainte qu'inspirent les esprits maléfiques.

\section{Selon la propriété}

Cinq (05) grands groupes de bois sacrés (Tableau 2) peuvent être définis suivant leurs propriétaires: les bois communautaires, les bois de quartier, les bois familiaux, les bois personnels et les bois des sociétés secrètes.

Les bois communautaires sont la propriété de toutes les communautés villageoises. Ils représentent $75,62 \%$ des bois sacrés (Figure 4). Cette catégorie de bois est présente chez tous les groupes ethniques possédant des bois sacrés (Tableau 2).

Les bois familiaux appartiennent à une famille précise, généralement celle des fondateurs du village. Ils sont rencontrés chez les Dafing, les Samo, les Bwaba et les Turka. $1,74 \%$ des bois sacrés forment ce groupe.

Chaque quartier dispose aussi d'au moins un bois sacré appelé bois sacré de quartier où les cultes sont rendus circonstanciellement aux ancêtres. On les retrouve en société Mossé, Dafing, Bwaba,
Samo et Turka. Ils représentent 18,40\% des bois sacrés.

Les bois sacrés personnels sont des bois qui appartiennent à un seul individu, généralement au chef de terre ou au chef de fétiche. Ils sont généralement rencontrés en société Samo, Bwaba, Gourousi et Turka et représentent $1,74 \%$ des bois inventoriés.

Les sociétés secrètes sont les premiers habitants qui occupaient le territoire Mossé actuel. Il s'agit notamment des Nioniossé. Cette société clanique Mossé dispose de bois sacrés avec des rituels compliqués et secrets dont aucun clan, aucune tribu n'a le droit d'y interférer. C'est cette herméticité qui justifie l'appellation de sociétés secrètes. Les bois des sociétés secrètes représentent $2,50 \%$ du total des bois.

\section{Principales fonctions des bois sacrés}

Les bois sacrés assurent à la population, plusieurs fonctions : écologique, cultuelle, socio-culturelle, magique et mixte (Figure 5 et 6).

Ils protègent plusieurs espèces végétales servant à l'alimentation, à des fins pharmaceutiques et pour de nombreux autres usages. Malgré les pressions croissantes, les bois sacrés abritent de nombreuses espèces végétales disparues dans les zones environnantes. Le bois sacré de Dierko dénommé Tizilinziido, dans la province du Yatenga, héberge une espèce rare en peuplement naturel, Manilkara multinervis (Bark.) Dubard qui a disparu du paysage, du sahel jusqu'au secteur nord soudanien. Un peuplement de Boswellia dalzielii Hutch. a été également observé au sein du bois Zombnoogo dans la province du Sanmatenga. Par ailleurs, les espèces telles que Boscia senegalensis (Pers.) Lam. ex Poir., Boscia angustifolia A. Rich., Celtis toka (Forssk.) Hepper \& Wood, Stereospermum kunthianum Cham., très rares dans les terroirs ont été rencontrées dans certains bois sacrés des secteurs strict sahélien, sub-sahélien et nord soudanien. Dans le secteur sud soudanien, les 
espèces comme Haematostaphis barteri Hook. f., Zanthoxylum zanthoxyloides (Lam.) Zepernick \& Timler, Luffa cylindrica L., Kaempferia aethiopica (Schweinf.) Benth., Hyphaene thebaica (L.) Mart., Gymnema sylvestre (Retz.) Schultes, Anchomanes difformis (B1.) Engl., Agave sisalana Perr., ont été seulement rencontrées dans les bois sacrés. Tous ces exemples confirment le rôle refuge des bois sacrés.

Les bois sacrés abritent les fétiches et/ou les divinités du village. Ces divinités associées aux créatures invisibles (génies et esprits) qui y vivent, serviraient d'intermédiaires entre la population et leurs ancêtres auxquels des cultes sont rendus. Des sacrifices rituels liés à la vie du village sont faits dans les lieux de culte à des fins diverses (demande de pluie, de bonne saison, de la santé de la population, etc.). $19,9 \%$ des bois sacrés assurent cette fonction (Figure 5).

Au Burkina Faso, les bois sacrés ont aussi un rôle commémoratif. Les sacrifices commémoratifs y sont faits à des moments précis de l'année, à des fins diverses (pluies, bonne saison, santé de la population).

Sur le plan socio-professionnel, les bois permettraient à la population d'avoir plus de chance dans les activités telles que le commerce, l'agriculture, l'élevage, les postes électifs ou nominatifs, la fonction public, etc.

A travers des sacrifices, des offrandes et des libations, les bois sacrés permettraient de restaurer la fertilité des hommes et des femmes. Il existe également des rituels de propitiations, des incantations et des invocations pour qu'une femme qui ne peut pas avoir d'enfant soit enceinte.

En dehors des tribunaux réguliers, c'est-à-dire la justice des hommes, il y a pour certains peuples indigènes, une autre justice à laquelle ils croient fermement parce qu'elle est infaillible et possède du divin. C'est ainsi que pour se défendre contre des accusations mensongères (vol, mensonge, litige sur une portion de terre, ...), il y a un serment d'incantation qu'on prête dans certains bois sacrés, devant les fétiches qui seraient à mesure de détecter les imposteurs.

Pendant l'époque coloniale, certains peuples indigènes Burkinabè nettement hostiles au recrutement militaire et aux grands travaux d'intérêt général ne sont pas restés indifférents face aux colons. Pour être sauvés $\mathrm{du}$ recrutement militaire, les jeunes ou leurs parents, faisaient des consultations auprès des nyctosophes, s'adonnaient à des bois sacrés ou se refugiaient dans la brousse.

Sur le plan médico-magique, les bois sacrés sont beaucoup sollicités pour les cures et les préventions de certaines maladies mystiquement transmises par des humains et esprits maléfiques, de maladies congénitales en apparence incurables, de maladies à diagnostics imprécis. Ils seraient aussi utilisés dans la protection contre les maléfices, la préservation de la paix sociale et pour se rendre invulnérable. $8,70 \%$ des bois assurent cette fonction (Figure 5).

Chez tous les groupes ethniques enquêtés, la population affirme que les bois sacrés pourraient leur aider à obtenir de l'eau en temps de sécheresse. Dans certaines localités, surtout celles où vivent toujours les Nionionsé indigènes, les bois sacrés, à travers des sacrifices et des offrandes, pourraient jouer un grand rôle dans la régulation de ces périodes de sécheresse.

Certains bois sacrés assurent à la fois une fonction écologique, socio-culturelle, cultuelle et magique (35,32\% des bois sacrés). Les autochtones tout comme les étrangers s'y rendent pour des besoins de toute nature. Ces types de bois sacrés sont rencontrés dans tous les groupes ethniques. 


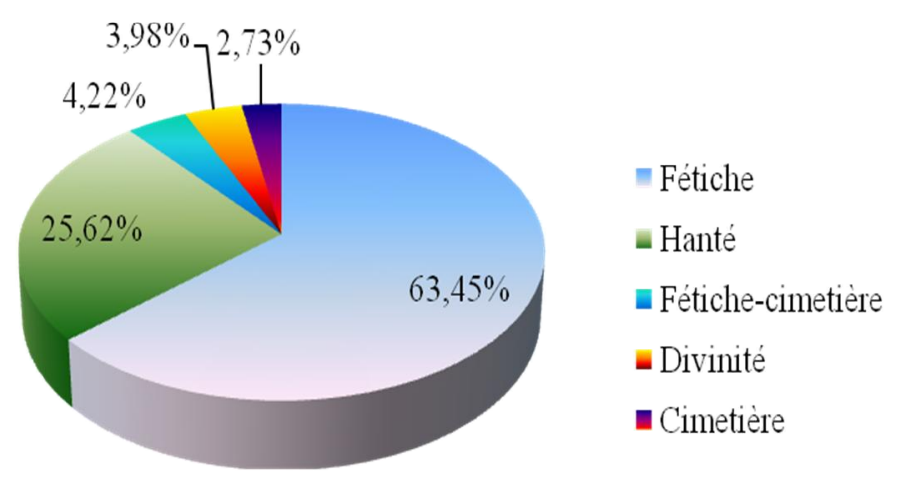

Figure 2: Proportions centésimales des bois sacrés selon leur incarnation.

Tableau 1: Types de bois sacrés et appartenance ethnique (selon l'incarnation).

\begin{tabular}{|c|c|}
\hline Types de bois sacré & Groupes ethniques \\
\hline Bois fétiches & $\begin{array}{c}\text { Tous les groupes ethniques excepté les Peulh, les } \\
\text { Touareg, les Bella et les Rimaibé }\end{array}$ \\
\hline Bois cimetières & Mossé, Fulsé, Gourmantché, Toussian, Yana, Bisa \\
\hline Bois fétiche-cimetières & Mossé, Fulsé, Gourmantché \\
\hline Bois hantés & $\begin{array}{l}\text { Mossé, Dafing, Fulsé, Gourousi, Bwaba, Bobo, } \\
\text { Gourmantché, Bolon, Yana, Bisa, Lobi, Birifor, } \\
\text { Gan, Djan, Pougouli, Dogossé, Turka, }\end{array}$ \\
\hline Bois de divinités & $\begin{array}{c}\text { Mossé, Gourmantché, Bwaba, Gourounsi, Samo, } \\
\text { Lobi, Birifor, Dagara, Gan, Pougouli }\end{array}$ \\
\hline
\end{tabular}

Tableau 2: Types de bois sacrés et appartenance ethnique (selon la propriété).

\begin{tabular}{lc}
\hline Types de bois sacré & Appartenance ethnique \\
\hline Bois sacrés communautaires & $\begin{array}{c}\text { Tous les groupes ethniques à l'exception des Peulh, } \\
\text { des Touareg, des Bella et des Rimaibé }\end{array}$ \\
\hline Bois de quartier & Mossé, Dafing, Samo, Bwaba, Turka \\
Bois familiaux & Samo, Dafing, Bwaba \\
Bois personnels & Samo, Bwaba, Gourounsi, Turka \\
Bois des sociétés secrètes & Mossé, Fulsé, Gourounsi \\
\hline
\end{tabular}




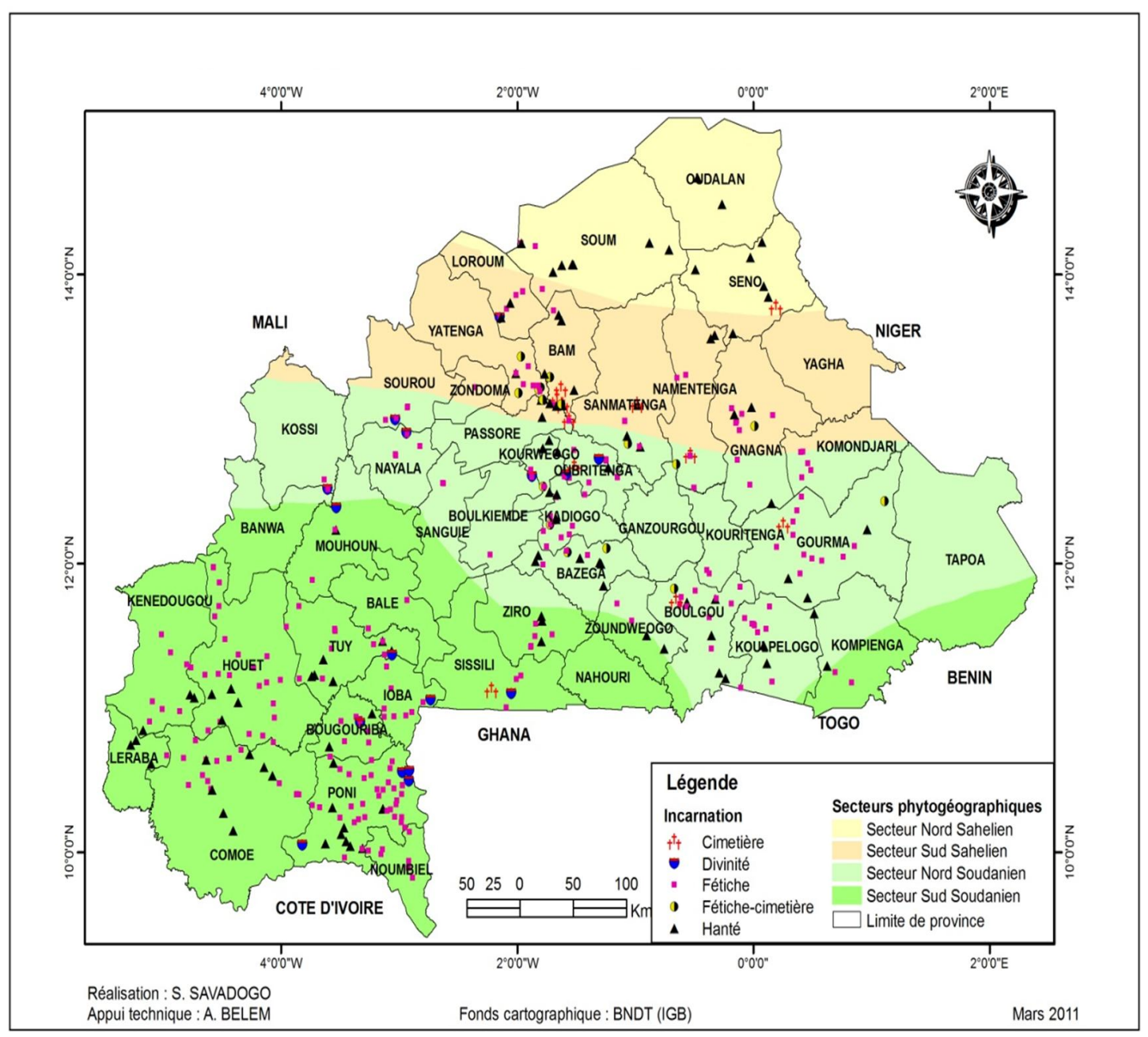

Figure 3: Carte de distribution des bois sacrés selon leur incarnation.

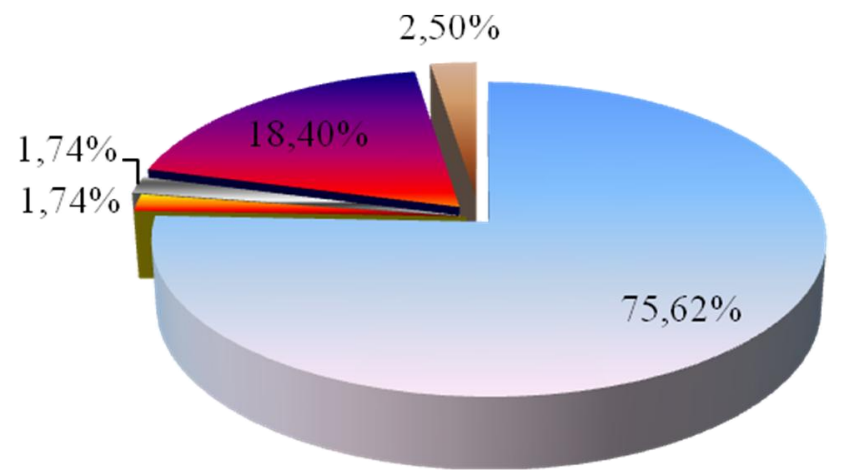

Communautaires

- Familiaux

$=$ Personnels

- Quartiers

- Sociétés secrêtes

Figure 4: Proportion centésimale des bois selon la propriété. 


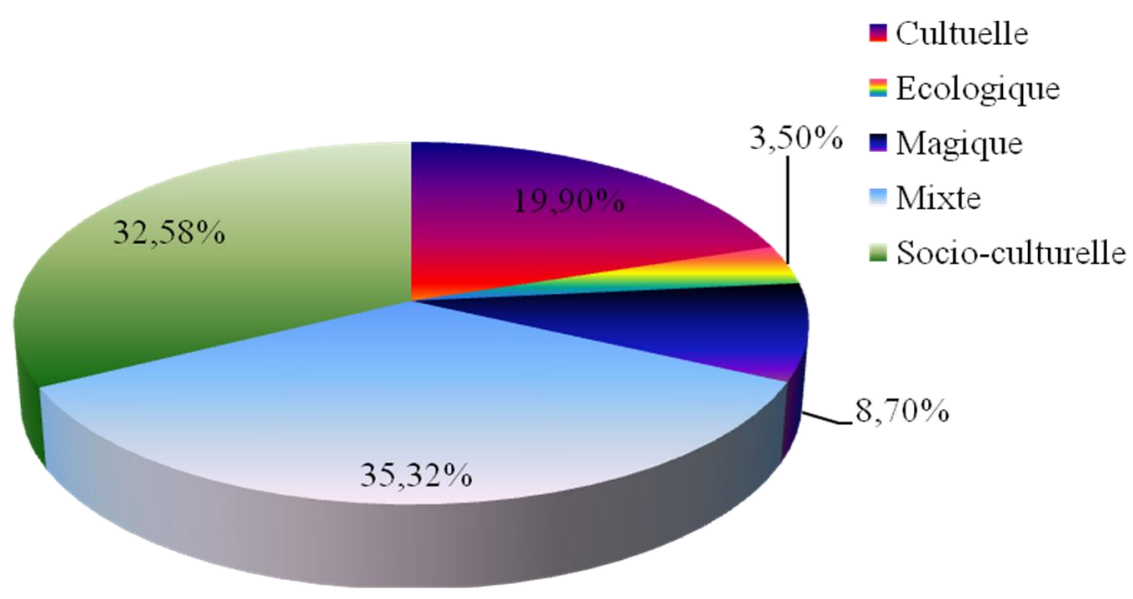

Figure 5: Répartition centésimale des principales fonctions des bois sacrés.

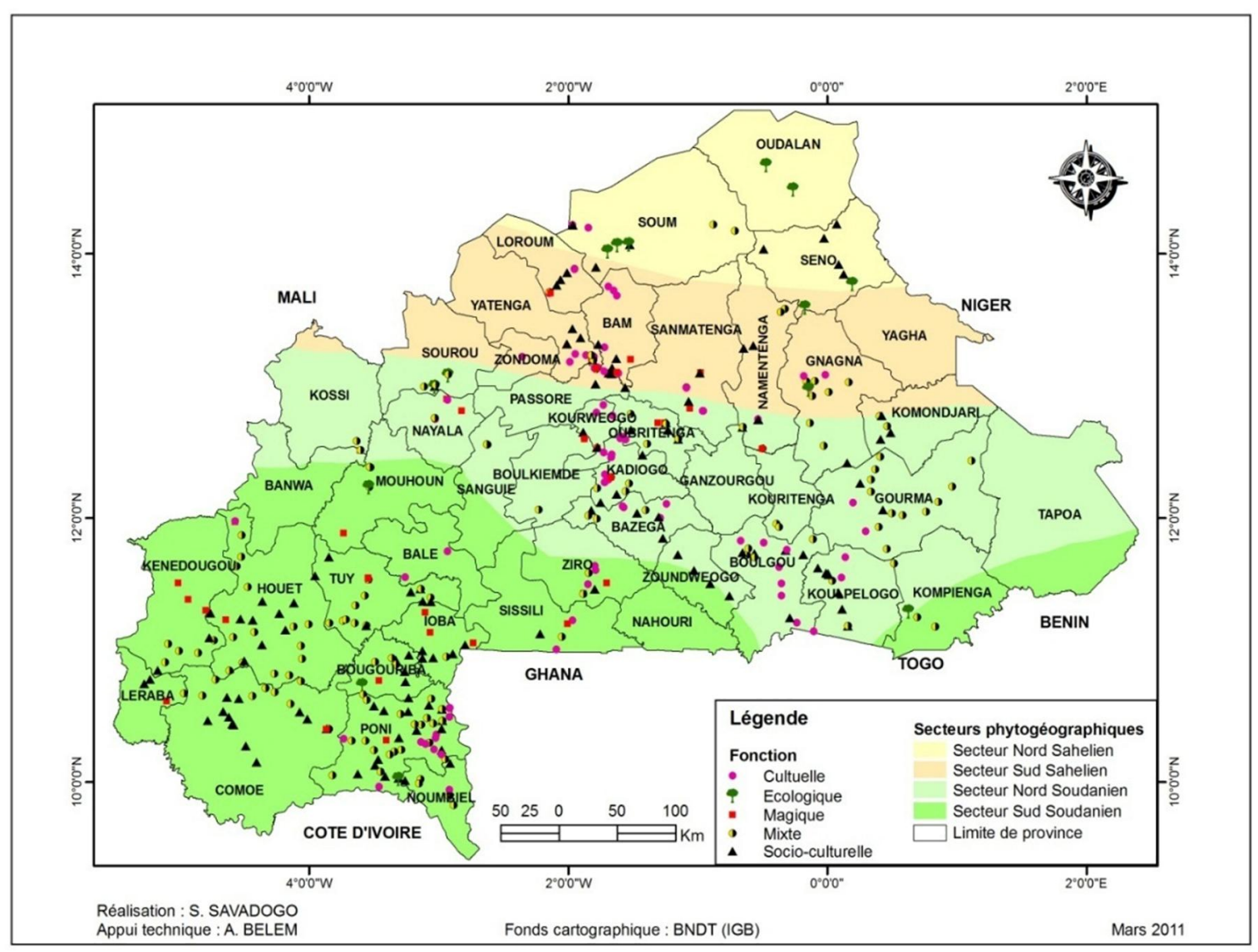

Figure 6: Carte de distribution des différents bois sacrés selon les fonctions qui leurs sont assignées. 


\section{DISCUSSION}

\section{Caractéristiques végétales des bois sacrés}

Les bois sacrés sont généralement des unités de végétation de petite taille, très souvent localisés au voisinage des habitats humains. Des études antérieures réalisées par certains auteurs ont montré des résultats similaires (Thiombiano, 2005; Traoré, 2007 ; Savadogo, 2008 ; Savadogo, 2013). On note une dominance des espèces telles Anogeissus leiocarpa, Combretum micranthum, Acacia erythrocalyx, Balanites aegyptiaca, Combretum nigricans dans la zone sahélienne et Khaya senegalensis, Anogeissus leiocarpa, Isoberlinia doka, Maranthes polyandra, Daniellia oliveri, Entada africana, Burkea africana et Monotes kerstingii dans la zone soudanienne (Savadogo, 2008; Savadogo et Thiombiano, 2010 ; Savadogo, 2013).

\section{Différents types de bois sacrés}

La classification des bois sacrés sur la base de leur représentation ou leur incarnation donne cinq (05) types principaux : les bois fétiches, les bois cimetières, les bois fétichecimetières, les bois de divinité et les bois hantés. Les bois fétiches prédominent l'ensemble des bois recensés et représentent $63,45 \%$ de l'effectif total. Cette prédominance est imputable à la volonté manifeste des autorités coutumières à préserver les acquis et les avantages de la religion ancestrale. Des études antérieures menées au Burkina Faso (Savadogo, 2013) ont montré des résultats similaires. La catégorisation des bois sacrés en bois cimetières, bois fétiches, et bois fétichecimetières est proche de celle effectuée par Kokou et Sokpon (2006) sur les forêts sacrées du couloir de Dahomey au Bénin.

Pour Boukepessi (2009), il est difficile d'adopter une typologie précise. Ainsi, a-t-il retenu plusieurs critères pour établir la typologie des bois sacrés du centre-Togo: suivant l'appartenance, suivant le sexe de la divinité, suivant le rapport de force, selon les pratiques, selon la superficie, selon l'effectif des divinités, etc.

Kaboré (2010), distingue cependant deux (02) catégories de lieux sacrés en société Gourmantché: les sites sacrés dangereux (abritant les génies malfaisants et les esprits de personnes « mortes par force » et les sites sacrés socialisés (ne présentant aucun danger).

Les bois cimetières et fétichecimetières bénéficient d'une autoprotection intégrale à cause de la crainte qu'inspirent les tombeaux de ceux qui y ont été enterrés, contrairement aux bois fétiches et aux bois de divinité jugés abstraits et seulement protégés par leur dépositaire.

Les bois hantés sont perçus dans l'imaginaire des peuples indigènes du Burkina Faso comme des fragments de végétation incarnant des créatures invisibles (Savadogo, 2008; Savadogo et al., 2011), le plus souvent irritant. Cette croyance au monde invisible susceptible de compromettre dangereusement la vie des humains, pourrait donc limiter les interventions humaines et préserver les espèces végétales de toute forme de destruction (coupe, mutilation, piétinement et arrachage par les animaux). Malan (2009) ne disait-il pas ceci: " même si la conservation n'est pas l'objectif premier de la sacralisation d'un site, la présence d'un Boson, redouté par la communauté confère une protection efficace aux ressources naturelles du lieu sacré ».

Les fétiches et les divinités par contre ne sont pas des objets pouvant susciter une crainte. Ce qui explique les cas de profanation des bois sacrés qui les abritent. Selon plusieurs autorités coutumières au nord du Burkina Faso, un bois sacré au vrai sens, n'a nécessairement pas besoin d'une protection de la population locale; il assure sa propre protection au mieux de ses potentialités spirituelles. Partant de cette conception, il nous semble logique de dire que seuls les bois cimetières, les bois fétiche-cimetières et les 
bois hantés sont les vrais bois sacrés car ils répondent tous au principe d'auto protection intégrale. Nous convenons donc avec Kaboré (2010) que seule la crainte qu'inspire un site le met à l'abri des interventions humaines.

\section{Fonctions et dimensions spirituelles des bois sacrés}

Comme l'ont souligné Kokou et Caballé (2000), Douillet (2001), OMPI (2002), Juhé-Beaulaton et al. (2005), Maldague (2005), Dhaila et Bhupendra (2007), Kokou et Kokutse (2007), Sukumara et Jeeva (2008), Boukepessi (2009), Kaboré (2010), Ehinnou Koutchika et al. (2013), les bois sacrés ont une grande importance socioculturelle. En effet, ils abritent des divinités, des fétiches, des cimetières et sont aussi des lieux d'initiation et de célébration de cérémonies rituelles. Selon Kokou et Kokutse (2007), les Ewé, peuple originaire du Tado au Togo pratiquent le Vaudou dans lequel la forêt et les espèces végétales tiennent une place importante, comme abris à de nombreuses divinités ou comme leurs symboles. Ces résultats sont également consolidés par ceux de Garcia et al. (2006) qui en Inde, décrivaient les forêts sacrées comme des fragments forestiers associés à un esprit, une divinité ou un temple et comme des écosystèmes préservés en raison des croyances et pratiques qui y sont associées. Beaucoup de fonctions sont assignées aux forêts sacrées comme la fécondité, la santé, la prospérité, la protection, la chasse, la prédiction des pluies, la justice. Les bois sacrés représentent des symboles identitaires des différentes sociétés (Cousin et Martineau, 2010). Ce sont des conservateurs des pratiques traditionnelles; ils offrent à la population des avantages multiples. En effet, $32,58 \%$ des bois inventoriés assurent une fonction socioculturelle, $19,9 \%$ une fonction magique, $3,5 \%$ une fonction écologique, $8,7 \%$ une fonction cultuelle. Les $35,32 \%$ des bois assurent à la fois une fonction magique, cultuelle, culturelle et écologique. Ces résultats sont en accord avec ceux de Sanou (2010) qui souligne que la tradition confère à la communauté villageoise son identité et sa personnalité et lui donne la capacité de relever les grands défis qui se présentent à chaque époque. Les propos de Kaboré (2010) ne dérogent également pas à toutes ces considérations socio-religieuses sus mentionnées. En effet, les populations locales, à travers des cultes voués aux ancêtres et aux puissances surnaturelles des bois sacrés, pensent que ces bois sont des pourvoyeurs de bien de toute nature. C'est au nom de ces pratiques traditionnelles que périodiquement les habitants se rendent sur les autels communautaires dans les forêts pour solliciter aux esprits de la brousse et à ceux des ancêtres des bénédictions en début de saison de pluies ou à la fin des récoltes pour les remercier. Ces croyances sont favorables à la conservation de la biodiversité dans les écosystèmes (Savadogo et al., 2011 ; Traoré et al., 2011).

\section{Conclusion}

Les investigations sur les bois sacrés ont permis de montrer leurs caractéristiques végétales. Les bois sacrés sont des ilots de végétations situés au voisinage des habitats humains et dominés par des espèces telles Acacia erythrocalyx, Anogeissus leiocarpa, Combretum micranthum, Combretum nigricans, Balanites aegyptiaca, Khaya senegalensis, , Isoberlinia doka, Maranthes polyandra, Daniellia oliveri, Entada africana, Burkea africana, Monotes kerstingii. ... Ils se présentent sous forme de steppes, de fourrés et ou de savanes arbustives en zone sahélienne et de savanes arbustives, de savanes boisées ou arborées, de forêts claires ou de galeries forestières en zone soudanienne. Par ailleurs, l'étude montre l'importance des bois sacrés pour les populations. Deux (02) critères principaux sont utilisés pour classer les bois sacrés. Ce sont: selon l'incarnation du bois 
sacré (bois fétiches, bois cimetières, bois fétiches-cimetières, bois de divinités et bois hantés) et selon le propriétaire (bois personnels, bois familiaux, bois de quartier, bois communautaires et bois de sociétés secrètes). De tous ces types de bois sacrés, seuls les bois cimetières, les bois fétichecimetières et les bois hantés sont intégralement protégés à cause de la crainte qu'ils suscitent à l'endroit de la population ; d'où la nécessité d'entreprendre des actions d'aménagement en leurs seins afin de mieux conserver la biodiversité.

\section{CONFLIT D'INTERETS} conflit d'intérêt.

Les auteurs déclarent qu'il n'y a aucun

\section{REMERCIEMENTS}

Nous remercions sincèrement les autorités coutumières des différents sites d'études pour leurs franches collaborations et leurs esprits de sympathie. Nos remerciements vont également à l'endroit du cartographe Monsieur Belem Alain pour son appui technique dans la réalisation des cartes de localisation de nos sites d'étude.

\section{REFERENCES}

Ali RKFM, Odjoubere J, Tente ABH., Sinsin AB. 2014. Caractérisation floristique et analyse des formes de pression sur les forêts sacrées ou communautaires de la Basse Vallée de l'Ouémé au Sud-Est du Bénin. Afrique Science, 10(2): 243-257. DOI : http://www.afriquescience.info

Bognounou O, Belem OM, Lamien N. 2001. Connaissances et pratiques traditionnelles pour une conservation de la biodiversité au Burkina Faso, 17 p.

Boukepessi T. 2009. Rôle socio-économique des bois sacrés du centre Togo, $12 \mathrm{p}$.

Cousin S, Martineau JL. 2010. Le festival, le bois sacré et l'UNESCO. Logiques politiques du tourisme culturel à Osogbo
(Nigeria). Cahiers d'Etudes Africaines, 139(1): 337-364. DOI : http://etudesafricaines.revues.org/18738

Dhaila-Adhikari S, Bhupendra SA. 2007. Veneration of a deity by restoration of sacred grove in a village Minar, Kumaun region of Uttarakhand: A case study. Journal of American science, 3(2): 1-5. DOI: http://www.jofamericanscience. org/journals/.../08-0255-Dhaila-Adhikari

Douillet R. 2001. L'arbre et la forêt, du symbolisme culturel à l'agonie programmée? $142 \mathrm{p}$.

Ehinnou Koutchika RI, Agbani PO, Sinsin B. 2013. Influence des perturbations anthropiques sur la biodiversité des bois sacrés du Centre Bénin. Int. J. Biol. Chem. Sci., 7(1): 306-318. DOI: http://dx.doi.org/10.4314/ijbcs.v7i1i.26

Fournier A. 2011. Consequences of wooded shrine rituals on vegetation conservation in West Africa: a case study from the Bwaba cultural area (West Burkina Faso). Biodivers. Conserv., 20: 18951910. DOI: http://www.iaees.org/ publications/journals/environsc/articles/2 013-2(1)/2013-2(1).asp

Garba A, Djima IT, Abdou L, Mahamane A. 2017. Caractérisation de la végétation ligneuse du bassin versant de la Maggia dans la commune rurale de Bagaroua (région de Tahoua). Int. J. Biol. Chem. Sci., 11(2): 571-584. DOI : https://dx.doi.org/10.4314/ijbcs.v11i2.4

Garcia C, Pascal JP, Kushalappa CG. 2006. Les forêts sacrées du Kadagu en Inde : écologie et religion. Bois et Forêts des Tropiques, 288(2): 5-13. DOI : http://bft.cirad.fr/cd/BFT_288_5-13.pdf

Houngnihin AR. 2005. Les mécanismes endogènes dans la problématique de l'environnement à Covè (Benin). $11^{\mathrm{e}}$ Assemblé générale du CODESRIA, Maputo (Mozambique), $19 \mathrm{p}$. 
Juhé-Beaulaton D, Dugast S, Tostain S, Kokou K, Alfieri C, Sanou A, Varissou S. 2005. Enjeu économique et sociaux autour des bois sacrés et la «conservation de la biodiversité». Atelier IFB, Paris «Usage locaux et dynamique de la biodiversité», $6 \mathrm{p}$.

Kaboré A. 2010. Brousse des uns, aire protégée des autres. Histoire du peuplement, perceptions de la nature et politique des aires protégées dans le Gourma burkinabè: l'exemple de la Réserve partielle de faune de Pama. Thèse de doctorat unique, Université de Genève, p. 389.

Kaboré A. 2010. Les stratégies communautaires d'adaptation au changement climatique: cas des bois sacrés dans le contexte socio-culturel moaga du Burkina Faso. Thèse de doctorat unique. Université d'AbomeyCalavi, Bénin, p. 235.

Kokou K, Caballé G. 2000. Les îlots forestiers de la plaine côtière Togolaise. Bois et Forêts des Tropiques, 263(1): 39-51. DOI : https://www.researchgate.net/.../ 237134780

Kokou K, Kokutse AD. 2007. Conservation de la biodiversité dans les forêts sacrées littorales du Togo. Bois et Forêt des Tropiques, 292: 59-72. DOI : https:/groupscientifictogo.files.wordpres s.com/2014/01/kokou.pdf

Kokou K, Sokpon N. 2006. Les forêts sacrées du couloir du Dahomey. Bois et Forêts des Tropiques, 288(2):15-23. DOI : http://www.abctaxa.be/...forets_sacrees_ du_couloir_du_dahomey

Langewiesche K. 2006. La forêt, les ancêtres et le marché. Perceptions locales de la forêt et de ses changements au NordBénin, Institut für Afrika-Kunde. Afrika Spectrum, $\quad$ 41(2): 221-248. DOI: https://doi.org/10.1515/IABI.2007. 019.
Malan DF. 2009. Religion traditionnelle et gestion durable des ressources floristiques en Côte d'Ivoire : le cas des ehotilé, riverains du parc national des îles ehotilé. Vertigo, 9(2): 1-11. DOI : $10.4000 /$ vertigo. 8661

Maldague M. 2005. Traité de gestion de l'environnement tropical. Développement intégré des régions tropicales: La place de la culture dans le développement. Fascicule I-1: Besoins essentiels de l'homme et développement intégré. Tome I, $20 \mathrm{p}$.

Matthieu S. 2010. Espaces politiques, espaces rituels : les bois sacrés de l'ouestCameroun. Presses de Sciences Po, 55(3) : 19-38. DOI : 10.3917/autr.055. 0019

Olohou EC, Ouinsavi C, Sokpon N. 2016. Facteur déterminant de la fragmentation du bloc forêt classée- forêts classées au sud Bénin. Journal of Applied Biosciences, 101: 9618-9633. DOI: http://dx.doi.org/10.4314/jab.v101i1.5

OMPI. 2002. Savoir traditionnelsTerminologie et définition. Troisième session du comité d'intergouvernemental de la propriété intellectuelle relative aux ressources génétiques, aux savoirs traditionnels et au folklore à Genève, 39 p.

Sanou BD. 2010. Aménagement des Forêts de Dinderesso et $d u$ Kou et Autoreprésentation des Communautés Villageoises Riveraines. Proposition d'une Méthode d'Actualisation des Coutumes pour une Gestion Durable des Ressources Naturelles. Centre Africain de recherche pour une pratique culturelle $\mathrm{du}$ Développement (CAD) : BoboDioulasso, Burkina Faso; 23.

Savadogo S. 2008. Etude de la flore et de la végétation des bois sacrés de la zone sub-sahélienne du Burkina Faso. 
Mémoire de DEA. Université de Ouagadougou, Burkina Faso, p. 65.

Savadogo S. 2013. Les bois sacrés du Burkina

Faso : diversité, structure, dimension spirituelle et mode de gestion de leurs ressources naturelles. Thèse de doctorat unique. Université de Ouagadougou, Burkina Faso, p. 226.

Savadogo S, Ouédraogo A, Thiombiano A. 2010. Perceptions, mode de gestion et végétation des bois sacrés au nord du Burkina Faso. Flora et Vegetatio Sudano-Sambesica, 13: 10-21. DOI: http://publikationen.ub.unifrankfurt.de/v olltexte/2008/5860

Savadogo S, Ouédraogo A, Thiombiano A. 2011. Diversité et enjeux de conservation des bois sacrés en société Mossi (Burkina Faso) face aux mutations socioculturelles actuelles. Int. J. Biol. Chem. Sci., 5(4): 1639-1658. DOI: 10.4314/ijbcs.v5i4.27

Savadogo S, Thiombiano A. 2010. Les bois sacrés et les forêts communautaires. In Atlas de la Biodiversité de l'Afrique de l'Ouest (Tome II), Thiombiano A, Kampmann D (eds). Frankfurt/Main : Ouagadougou, Burkina Faso ; 378-385.

Seydou N, Mohamed MC, Ndiaye O, Malaïny D. 2017. Influence de la flore ligneuse associée dans la production des parcs à Anacardium occidentale L. dans la communauté rurale de Djibanar
(Casamance/Sénégal). Int. J. Biol. Chem. Sci., 11(2): 585-596. DOI: https://dx.doi.org/10.4314/ijbcs.v11i2.5 Sukumaran S, Jeeva S, 2008. A floristic study on miniature sacred forests at Agastheeshwaram, southern peninsular India. Eur Asian Journal of Biosciences, 2: 66-72. DOI : http://www.ejobios.com/ content $/ 2 / 8 / 66-72$

Thiombiano A. 2005. Les Combretaceae du Burkina Faso: Taxonomie, écologie, dynamique et régénération des espèces. Thèse de doctorat d'Etat. Université de Ouagadougou, Burkina Faso, p. 290.

Traoré L. 2007. Bois sacrés, étude de la végétation ligneuse, mode de gestion dans les terroirs de Tiankuy, région de la boucle du Mouhoun. Mémoire de DEA. Université de Ouagadougou, Burkina Faso, p. 50.

Traoré L, Ouédraogo I, Ouédraogo A, Thiombiano A. 2011. Perceptions, usages et vulnérabilité des ressources végétales ligneuses dans le Sud-Ouest du Burkina Faso. Int. J. Biol. Chem. Sci., 5(1): 258-278. DOI : http://dx.doi.org/10.4314/ijbcs.v5i1.6810 3

WWF-France 2003. Ecologie et spiritualité: Vers un réenchantement du monde, $21 \mathrm{p}$. $<$ http: //www.wwf.fr/content download /1120/5139/version/1/file/> 